\title{
Optimizing Network Resource Sharing in Grids
}

\author{
L. Marchal, P. Vicat-blanc Primet, Y. Robert, and J. Zeng \\ LIP, UMR CNRS-ENS Lyon-INRIA-UCB Lyon 5668 \\ École Normale Supérieure de Lyon, France
}

\begin{abstract}
While grid computing reaches further to geographically separated clusters, data warehouses, and disks, it poses demanding requirements on end-to-end performance guarantee. Its pre-defined destinations and service criteria ease the performance control; however, expensive resources and equipments used by grid applications determine that optimal resource sharing, especially at network access points, is critical. From the resource reservation perspective, this article looks at communication resources shared by grid sites. Two resource request scenarios have been identified, aiming at optimizing the request accept rate and resource utilization. The optimization problems, proven NP-complete, are then solved by heuristic algorithms. Simulation results, aside from showing satisfying results, illustrate the pros and cons of each algorithm.

Keywords: grid computing, communication resource, resource sharing, optimization.
\end{abstract}

\section{INTRODUCTION}

Grid computing is a promising technology that brings together geographically distributed resources. Grids aggregate a large collection of resources(e.g., computing, communication, storage, information, etc.) to build a very high-performance computing environment for dataintensive or computing-intensive applications [1].

Grid applications, such as distance visualization, bulk data transfer, and high-end collaborative environment, have diverse and demanding performance requirements [2]; for instance, the coordinate management of network, storage, and computing resources, dynamically control over QoS and application behaviors, and advance resource reservation. Analyses [3] have shown that grids demand broad service quality, such as guaranteed delivery of huge data files [4], TCP throughput predictability, and data delivery stability.

The underlying communication infrastructure of grids, moreover, is a complex interconnection of LANs and WANs that introduces potential bottlenecks and varying performance characteristics [5]. For instance, the interface between LAN and WAN, considering grid sites may generate large flows through their gigabit interfaces, introduces resource sharing bottleneck. Herein, provisioning end-to-end services with known and knowable characteristics of grids, which spans multiple administrative and technological domains, is critical.
An approach to tackle this problem is network resource reservation [6]. While computational/storage resource sharing/scheduling has been intensively investigated for grids [7]-[10] during the past years, surfacing is the idea of incorporating network/communication resource management into grid environments.

Based on the Grid 5000 project [11], an experimental grid platform gathering 5000 processors over eight sites geographically distributed in France, this article centers on network resource sharing. The rest of the article is organized as follows. Section II gives the system model and defines optimization problems for network resource sharing. Section III proves that the optimization problem is NP-complete. Heuristics and simulation results are given in section IV and section V, respectively. SectionVI presents related work. Finally, the article concludes in section VII.

\section{System Model AND PROBlem Definition}

Derived from physical configuration of the Grid5000 network, the system model is a collection of LANs (that is, grid sites) interconnected over a well-provisioned WAN. They are connected through IP routers. The grid network middleware carries out the network resource reservation task and communicates with grid applications. The network core is assumed to have ample communication resources [12]. Here, the aggregated capacity of a LAN is larger than the capacity of its access point (i.e., the router), and the capacity of the network core is larger than the aggregated capacity of all access points.

Given a set of resource requests, one can separate grid sites into ingress and egress points: where the traffic requires to enter the network from, is the ingress point, and where the traffic requires to leave the network from, is the egress point. These points at the network edge, as depicted in Fig. 1, are where resource sharing bottlenecks present.

\section{A. Resource requests}

Resource requests, corresponding to different application scenarios, can be long-lived or short-lived. The difference is that short-lived requests have time windows specified, as detailed below. 


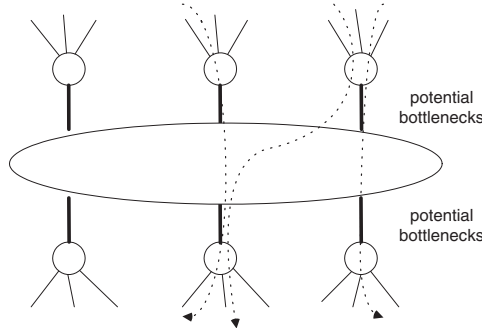

Fig. 1. The system model that shows ingress and egress points of a network as potential bottlenecks.

Given the notation as follows:

- a set of requests $\mathcal{R}=\left\{r_{1}, r_{2}, \ldots, r_{K}\right\}$, with $b w(r)$ as the bandwidth demanded by request $r \in \mathcal{R}$.

- a set of ingress points $\mathcal{I}=\left\{i_{1}, i_{2}, \ldots, i_{M}\right\}$, with $B_{i n}(i)$ as the capacity (i.e., bandwidth) of ingress point $i \in \mathcal{I}$.

- a set of egress points $\mathcal{E}=\left\{e_{1}, e_{2}, \ldots, e_{N}\right\}$, with $B_{\text {out }}(e)$ as the capacity (i.e., bandwidth) of egress point $e \in \mathcal{E}$.

For each request $r \in \mathcal{R}$, resource sharing constraints are stated as:

$$
\begin{aligned}
& \forall i \in \mathcal{I}, \quad \sum_{r \in \mathcal{R}, \text { ingress }(r)=i} b w(r) \leqslant B_{\text {in }}(i) \\
& \forall e \in \mathcal{E}, \quad \sum_{r \in \mathcal{R}, \text { egress }(r)=e} b w(r) \leqslant B_{\text {out }}(e)
\end{aligned}
$$

where $\operatorname{ingress}(r) \in \mathcal{I}$ and egress $(r) \in \mathcal{E}$ are the ingress and egress point of request $r$, respectively.

For short-lived requests, more parameters are introduced as:

- each request $r \in \mathcal{R}$ has a starting time $t_{s}(r)$ and a finishing time $t_{f}(r)$. The time window of request $r$ is then $\left[t_{s}(r), t_{f}(r)\right]$.

- Each request $r \in \mathcal{R}$ has its volume $\operatorname{vol}(r)$ specified either in Bytes or other meaningful units.

If request $r$ is accepted at time $\sigma(r)=t$, both points of ingress $(r)$ and egress $(r)$ devote a fraction of their capacity, that is, $b w(r)$, to request $r$ from time $t$ to time $\tau(t)=t+\frac{v o l(r)}{b w(r)}$. Obviously, the scheduled window of $[\sigma(r), \tau(r)]$ must be included in the time window of $\left[t_{s}(r), t_{f}(r)\right]$ for all requests $r \in \mathcal{R}$, that is,

$$
\forall r \in \mathcal{R}, \quad t_{s}(r) \leqslant \sigma(r)<\tau(r) \leqslant t_{f}(r)
$$

Applying to the short-lived requests with scheduled time window $[\sigma(r), \tau(r)]$, the resource constraints (1) are now restated as:

$$
\begin{aligned}
& \forall t, \forall i \in \mathcal{I}, \sum_{\substack{r \in \mathcal{R}, \text { ingress }(r)=i, \sigma(r) \leqslant t<\tau(r)}} b w(r) \leqslant B_{\text {in }}(i) \\
& \forall t, \forall e \in \mathcal{E}, \sum_{\substack{r \in \mathcal{R}, \sigma(r) \leqslant t<\tau(r) \\
\sigma(r)=e,}} b w(r) \leqslant B_{\text {out }}(e)
\end{aligned}
$$

\section{B. Optimization objectives}

To formulate the optimization problem, $x_{k}$ is defined as a boolean variable; it is equal to 1 if and only if request $r_{k}$ is accepted. Provided with different types of requests and constraints specified in subsection II-A, two optimization objectives are given as below:

a) MAX-Requests : Under the constraints in (1) or (2), one may maximize the ratio of the number of accepted requests to that of total requests. The objective function, referred to as MAX-REQUESTS, is:

$$
\text { MAXIMize } \sum_{k=1}^{K} x_{k}
$$

b) Resource-Util: Under the same constraints, one may maximize the resource utilization ratio, that is, the ratio of granted resources to total resources. The objective function, referred to as RESOURCE-UTIL, is:

$$
\text { MAXimize } \frac{\sum_{k=1}^{K} x_{k} \cdot b w\left(r_{k}\right)}{\frac{1}{2}\left(\sum_{i=1}^{M} B_{\text {in }}^{\text {scaled }}(i)+\sum_{e=1}^{N} B_{\text {out }}^{\text {scaled }}(e)\right)},
$$

where the numerator $\sum_{k=1}^{K} x_{k} . b w\left(r_{k}\right)$ is the total bandwidth that has been assigned to requests. Since one bandwidth request is counted twice, that is, at both ingress and egress points, a factor of $1 / 2$ is used to "stretch" the utilization value to 1 .

Furthermore, defined as

$$
B_{\text {in }}^{\text {scaled }}(i)=\min \left(B_{\text {in }}(i), \sum_{r \in \mathcal{R}, \text { ingress }(r)=i} b w(r)\right)
$$

and

$$
B_{\text {out }}^{\text {scaled }}(e)=\min \left(B_{\text {out }}(e), \sum_{r \in \mathcal{R}, \text { egress }(r)=e} b w(r)\right),
$$

$B_{\text {in }}^{\text {scaled }}(i)$ and $B_{\text {out }}^{\text {scaled }}(e)$ are adopted to rule out the possibility where one access point has no requests at all; thus, the capacity of this point shall be excluded when calculating resource utilization.

\section{COMPleXity ANALYsis}

Since the linear program for MAX-REQUESTS involves integer (boolean) variables, there is little hope that an optimal solution could be computed in a polynomial time. Indeed, optimization problems MAX-REQUeSTS and RESOURCE-UTIL both turn out to be NP-complete, as shown in the rest of the section.

The decision problem associated to the MAXREQUESTS problem is the following:

Definition 1 (MAX-REQUests-DeC): Given a problem-platform pair $(\mathcal{R}, \mathcal{I}, \mathcal{E})$ and a bound $Z$ on the number of request to satisfy, is there a solution to the 
MAX-REQUESTS linear program such that $\sum_{k=1}^{K} x_{k} \geqslant$ $Z$ ?

Theorem 1: MAX-REQUESTS-DEC is NP-complete.

Proof: Clearly, MAX-REQUESTS-DeC belongs to NP; we prove its completeness by reduction from 2PARTITION, a well-known NP-complete problem [13]. Consider an instance $B_{1}$ of 2-PARTITION: given $n$ integers $\left\{a_{1}, a_{2}, \ldots, a_{n}\right\}$, is there a subset $I$ of indices such that $\sum_{i \in I} a_{i}=\sum_{i \notin I} a_{i}$ ? Let $S=\sum_{i=1}^{n} a_{i}$ and assume, without loss of generality, that $1 \leqslant a_{i} \leqslant S / 2$ for $1 \leqslant i \leqslant n$. We build the following instance $B_{2}$ of MAX-REQUESTS-DEC:

- There are $K=2 n$ requests in $\mathcal{R}$, and $b w\left(r_{k}\right)=$ $b w\left(r_{k+n}\right)=a_{k}$ for $1 \leqslant k \leqslant n$.

- There are $M=2$ ingress points and $N=n$ egress points. For ingress points we let $B_{i n}\left(i_{1}\right)=$ $B_{i n}\left(i_{2}\right)=S / 2$. For egress points we let $B_{\text {out }}\left(e_{k}\right)=a_{k}, 1 \leqslant k \leqslant n$.

- We let ingress $\left(r_{k}\right)=i_{1}$, ingress $\left(r_{k+n}\right)=i_{2}$, and $\operatorname{egress}\left(r_{k}\right)=\operatorname{egress}\left(r_{k+n}\right)=e_{k}$ for $1 \leqslant k \leqslant n$.

- Finally, we let $Z=n$. In other words, we aim at satisfying half of the requests.

The size of $B_{2}$ is polynomial (and even linear) in the size $B_{1}$. We have to show that $B_{1}$ has a solution if and only if $B_{2}$ has a solution.

Assume first that $B_{1}$ has a solution. Let $I$ be the subset of $\{1,2, \ldots, n\}$ such that $\sum_{i \in I} a_{i}=\sum_{i \notin I} a_{i}=S / 2$. We claim that we can satisfy the $|I|$ requests $r_{k}, k \in I$ together with the $n-|I|$ requests $r_{k+n}, k \notin I$, thereby achieving the desired bound $Z=n$. Indeed, we schedule the first $|I|$ request from ingress point $i_{1}$, and the remaining $n-|I|$ ones from $i_{2}$, without exceeding their capacity $B_{i n}\left(i_{1}\right)=B_{i n}\left(i_{2}\right)=S / 2$. Egress point $e_{k}$ is used either for request $r_{k}$ if $k \in I$, or for request $r_{k+n}$ if $k \notin I$; in either case, $B_{\text {out }}\left(e_{k}\right)=a_{k}$ is equal to the requested bandwidth for the request.

Conversely, assume now that $B_{2}$ has a solution. Let $I$ be the set of indices $k$ such that $r_{k}$ is satisfied and $1 \leqslant k \leqslant n$. Similarly, let $J$ be the set of indices such that $r_{k+n}$ is satisfied and $1 \leqslant k \leqslant n$. Because the capacity of egress point $e_{k}$ is $B_{\text {out }}\left(e_{k}\right)=a_{k}, I$ and $J$ must be disjoint: if they shared an index, the capacity of the corresponding egress point would need to be as twice larger as it is. Because the bound $Z=n$ is achieved, we have $|I|+|J| \geqslant n$. We deduce that $I$ and $J$ form a partition of $\{1,2, \ldots, n\}$. We have $\sum_{k \in I} a_{k} \leqslant S / 2$ because the capacity of ingress point $i_{1}$ is not exceeded, and $\sum_{k \in J} a_{k} \leqslant S / 2$ because the capacity of ingress point $i_{2}$ is not exceeded. But $I \cup J=\{1,2, \ldots, n\}$ and $\sum_{k=1}^{n}=S$, hence $\sum_{k \in I} a_{k}=\sum_{k \notin I} a_{k}=S / 2$. We have found a solution to $B_{1}$.
Proposition 1: The decision problem associated to RESOURCE-UTIL is NP-complete.

For the sake of brevity, we do not formally state the decision problem associated to RESOURCE-UTIL, and we refer to [14] for a proof of Proposition 1. Interestingly, it is also shown in [14] that MAX-REQUESTS-DEC problem remains NP-complete in the case of an uniform network (all ingress/egress capacities are equal); only in the (very unlikely in practice) case of an uniform network and uniform requests (all request bandwidths are equal) can the optimal solution of MAX-REQUESTS be computed in a polynomial time.

Since the problems defined in Section II have been proven to be NP-complete, solutions are pursued with heuristics.

\section{POLYNOMIAL HEURISTICS AND SIMULATIONS FOR LONG-LIVED REQUESTS}

Three polynomial heuristics are proposed for both optimization objectives MAX-REQUESTS and RESOURCEUTIL.

\section{A. Growing the set of accepted requests}

Based on classical greedy algorithm where requests are accepted until there are no more available resources, MAXREQ-SIMPLE sorts requests by bandwidth in a nondecreasing order (ties are broken arbitrarily). A request is accepted if and only if its requested bandwidth does not exceed the available capacity of both ingress and egress points. The pseudo code of the algorithm [14] is omitted here.

MAXREQ-REFINED refines the previous procedure, by accepting the request that leaves the maximum amount of resources to others. Take request $r_{k}$ as an example. Let $i=\operatorname{ingress}\left(r_{k}\right)$, and let alloc_ingress $(i)$ be bandwidth of point $i$ which has been taken by accepted requests (initially alloc_ingress $(i)=0$ ). By calculating the utilization ratio of ingress point $i$, that is, $\frac{\text { alloc_ingress }(i)+b w(k)}{B_{\text {in }}(i)}$, and that of the corresponding egress point, the request that minimizes this ratio is accepted. The details of the algorithm can be referred to [14].

\section{B. Peeling off the set of original requests}

Starting from the whole set of requests (i.e., the set of accepted requests $\mathcal{A}=\mathcal{R}$ ), MAXUsePEELING "peels off" certain requests until a solution meeting all resource constraints is found. Given the set of requests, an occupancy ratio defined as $\operatorname{ratio}(i)=\frac{\sum_{r \in \mathcal{A}, \text { ingress }(r)=i} b w(r)}{B_{i n}(i)}$ is calculated for all access points. If all ratios are smaller than 1, all requests are accepted. Otherwise, among requests whose ingress and egress points both have 
their occupancy ratio bigger than 1 , the one that helps decrease the ratio the most is peeled off; requests, either of whose ingress or egress points has a ratio bigger than 1 , are scanned through in a similar manner. The heuristic is detailed in [14].

\section{Simulation settings}

It is assumed that there are 50 ingress and egress points, respectively. The capacity of each point is randomly chosen as either $1 \mathrm{~Gb} / \mathrm{s}$ or $10 \mathrm{~Gb} / \mathrm{s}$. Requests may occur between any pair of different points, and its bandwidth request is randomly chosen from a set of values: $\{10 \mathrm{MB} / \mathrm{s}, 20 \mathrm{MB} / \mathrm{s}, \ldots, 90 \mathrm{MB} / \mathrm{s}, 100 \mathrm{MB} / \mathrm{s}, 200 \mathrm{MB} / \mathrm{s}$, $\ldots, 900 \mathrm{MB} / \mathrm{s}, 1000 \mathrm{MB} / \mathrm{s}\}$. The number of requests is determined by the system load, which is defined as the ratio of the sum of demanded bandwidth and the sum of available bandwidth in the system:

$$
\text { load }=\frac{\sum_{r \in \mathcal{R}} b w(r)}{\frac{1}{2}\left(\sum_{i \in \mathcal{I}} B_{\text {in }}(i)+\sum_{e \in \mathcal{E}} B_{\text {out }}(e)\right)}
$$

\section{Simulation results and discussion}

The simulation results for long-lived requests are illustrated in Figure 2.
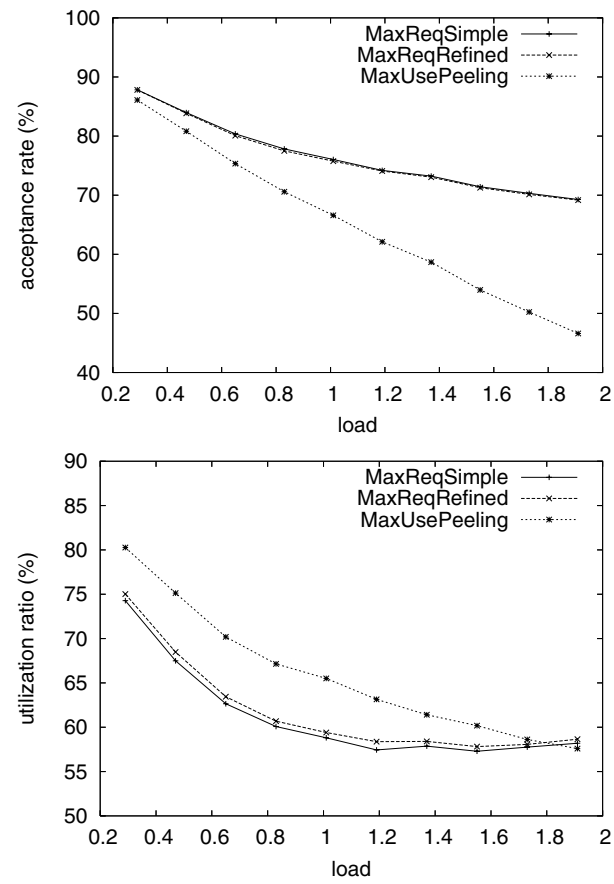

Fig. 2. Comparison of the heuristics for long-lived requests.

Obviously, MAXREQ-Simple and MAXREQREFINED, aiming at accepting as many requests as possible, outperforms MAXUSEPEeLING with respect to the accept rate. And MAXUsePeEling achieves better utilization ratio because it targets at optimizing the resource utilization. The original purposes of these heuristics have been met.

One may argue that none of the strategies reaches $100 \%$ acceptance rate or utilization ratio. The reason is that randomly generated requests in the article are not uniformly distributed among access points. It is not rare that certain point are heavily loaded, and certain points are not. The plotted accept rate and utilization ratio, which are more than $50 \%$, are actually rather satisfying.

\section{POLYNOMIAL HeURISTICS AND SIMUlations FOR SHORT-LIVED REQUESTS}

As illustrated in subsection II-A, if request $r$ with time window $\left[t_{s}(r), t_{f}(r)\right]$ is accepted at time $\sigma(r)=t$, a fraction of system capacity, that is, $b w(r)$, is scheduled to request $r$ from time $t$ to time $\tau(t)=t+\frac{v o l(r)}{b w(r)}$. Assume that time constraints are rigid, that is, $\sigma(r)=t_{s}(r)$ and $\tau(r)=t_{f}(r)$. Requests are then accepted or rejected as they are.

Note that, sharing the same complexity characteristics with long-lived ones, resource sharing optimization for short-lived requests is also NP-complete.

\section{A. FIFO}

Scheduling requests in a "first come first serve" manner, FIFO heuristic accepts requests in the order of their starting times. If several requests happen to have the same starting time, the request demanding the smallest bandwidth is scheduled first.

\section{B. Time window decomposition}

With rigid time windows, pre-defined starting and finishing times are used as reference points for resource scheduling. As depicted in Figure 3, these time points naturally form time intervals within which no request starts or stops; thus heuristics for long-lived requests in Section IV can be applied. Given intervals $\left[t_{0}, t_{1}\right],\left[t_{1}, t_{2}\right], \ldots,\left[t_{i-1}, i_{N}\right]$, therefore, for each $t_{i}$, there exists a request $r$ such that $t_{s}(r)=t_{i}$ or $t_{f}(r)=$ $t_{i}$. The greedy strategies proposed in Section IV are then applied to each time-interval, with two situations explained in the following paragraphs.

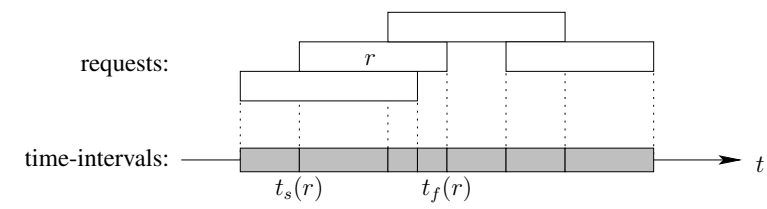

Fig. 3. Decomposition of requests with time windows. 
For a request that spreads over multiple time intervals, first, if it gets rejected in its first time interval, it will be discarded permanently; second, if it gets accepted in its first time interval, it shall be granted certain priority when competing with other requests in its future time intervals.

Taking the duration of a request and the scheduling decisions in previous time intervals into consideration, a priority factor is used to represents the importance of scheduling request $r$ on a given time-interval. Assume requests in time-intervals $\left[t_{0}, t_{1}\right],\left[t_{1}, t_{2}\right], \ldots,\left[t_{i-1}, t_{i}\right]$ have been scheduled, At the interval of $\left[t_{i}, t_{i+1}\right]$, the priority factor is defined as the sum of the time already allocated to the request $\left(t_{i}-t_{s}(r)\right)$ and the duration of the current interval $\left(t_{i}-t_{i-1}\right)$ over the total request duration, that is,

$$
\operatorname{priority}\left(r,\left[t_{i}, t_{i+1}\right]\right)=\frac{t_{i+1}-t_{s}(r)}{t_{f}(r)-t_{s}(r)}
$$

The cost factor defined in the MAXREQ-REFINED heuristic for long-lived requests, is then refined as follows:

$$
\operatorname{cost}\left(r,\left[t_{i}, t_{i+1}\right]\right)=\frac{b w(r)}{b_{\min } \times \operatorname{priority}\left(r,\left[t_{i}, t_{i+1}\right]\right)}
$$

where $b_{\min }=\min \left\{B_{\text {in }}(\right.$ ingress $\left.(r)), B_{\text {out }}(\operatorname{egress}(r))\right\}$

By adopting this cost factor, for requests with the same starting time, a higher priority is given to requests with smaller duration; it maximizes the accepted number of requests. For requests within the same time interval, a higher priority is given to requests that have been granted more resources. The complete heuristic CUMULATEDSLOTS is detailed in [14].

Following the same time window decomposition technique, two variants of the previous heuristic, that is, MINBW-SLOTS and MINVOL-SLOTS, are proposed with re-defined cost factor $\operatorname{cost}\left(r,\left[t_{i}, t_{i+1}\right)\right]=b w(r)$ and $\operatorname{cost}\left(r,\left[t_{i}, t_{i+1}\right)\right]=\operatorname{vol}(r)$, respectively.

\section{Simulation settings}

There are 10 ingress and 10 egress points, respectively, with a capacity of $1 \mathrm{~GB} / \mathrm{s}$. Ingress/egress points and demanded bandwidth are generated as in subsection IVC. Moreover, the requested volume is randomly chosen between 100GB and 1TB. Following the Poisson distribution, the request arrivals determine the system load similarly as in subsection IV-C.

\section{Simulation results and discussion}

As illustrated in Figure 4, first, FIFO shows poor performance on both accept rate and utilization ratio. The fact that FIFO lets requests block each other indicates that selectively reject is an important step towards good performance. Second, MINVOL-SLOTS does not perform as well as MINBW-SLOTS and CUMULATEDSLOTS. In fact, accepting a request with the minimum volume may not always be a good decision. If the time window is small, the request will likely take the majority of the bandwidth; this lowers the value of the accept rate and thus the utilization ratio. Last, CUMULATEDSLOTS and MINBW-SLOTS have very close performance. CUMULATED-SLOTS should have good performance because its decision is made based on both demanded bandwidth and resource reservation in the past; it prevents a request from being rejected in the late stage of its time window. MINBW-SLOTS accepts the requests with smaller bandwidth requirements; these requests are unlikely to be rejected later, unless other requests with small bandwidth demand surges at one point. Under some circumstances, MinBW-SLOTS performs as well as Cumulated-Slots, even without resource reservation history.
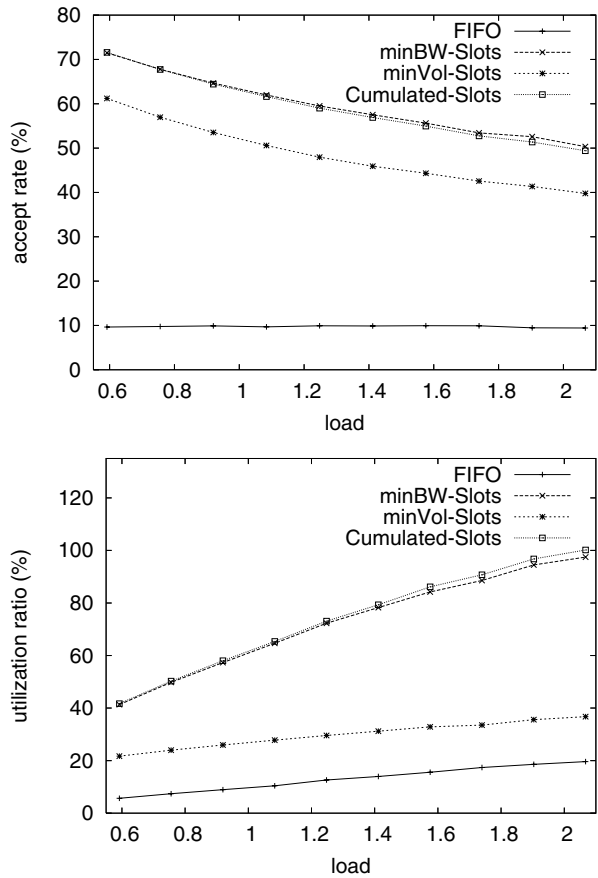

Fig. 4. Comparison of the heuristics according to two different metrics

\section{RELATED WORK}

Admission control mechanisms in IP networks are well-developed [15]. They have been mostly done at the ingress points of the network edge, or is closely coupled with feasible path search. The work in this article, however, looks at both access points where the traffic enters and leaves the network. Besides, the specific network topology studied in this article does not pose significant requirements on routing. 
Studying control mechanisms at network edge, this work is in line with the Internet philosophy of pushing the complexity to the network edge. On the perspective of resource scheduling, it pursues solutions based on the idea of what enters the network shall be able to leave the network, that is, the idea of avoiding potential packet drop within the network. This idea of "globally max-min fair" was investigated in Network Border Patrol [16], a core-stateless congestion avoidance mechanism.

Advance reservation for grids has also been under intensive study. The Globus Architecture for Reservation and Allocation (GARA) provides advance reservations and end-to-end management for QoS on different type of resources (network, storage and computing) [6]. A QoS architecture that combines resource reservation and application adaptation has been proposed. The work in this article fits in this context, but further explores the optimization on network resource sharing, based on a specific topology.

The advance reservation problem has also been defined and investigated in [17]. Although both targeting at resource requests with starting and finishing time limits, the work in this article looks at optimal resource sharing over a network with resource bottlenecks occurring at the edge, rather than investigating on impacts of the percentage of book-ahead periods and that of malleable reservations on the system.

\section{CONCLUSIONS}

Network resource sharing in grids has been investigated in this article. With bottlenecks presented at the network edge, network resources are reserved based on the concept of what enters the network shall be able to leave the network. For both long-lived and short-lived requests, optimization objectives with respect to request accept rate and resource utilization are pursued. Proven to be NP-complete, the optimization problems are solved with heuristics. The heuristic algorithms are studied and compared by simulations.

Resource sharing optimization studied in this article can be extended to other similar environments, for example, community overlay networks. The resource sharing strategies can be carried out either in a centralized or distributed manner, depending on network management implementations. Future work will be continued in the direction of reliving tentative hot spots in the network, that is, ingress/egress points that are heavily demanded, and in the direction of real-time resource reservation.

\section{ACKNOWLEDGMENT}

This work has been funded by the French ministry of Education and Research, INRIA, and CNRS, via ACI
GRID's Grid5000 project and GRIPPS project.

\section{REFERENCES}

[1] I. Foster, The Grid 2: Blueprint for a New Computing Infrastructure. Morgan Kaufmann, 2004.

[2] V. Sander, W. Allcock, C. Pham, I. Monga, P. Padala, M. Tana, and F. Travostino. (2003, June) Networking issues of grid infrastructures. Grid working draft, Grid High Performance Networking Research Group (GHPN-RG), Global GRID Forum. [Online]. Available: http://forge.gridforum.org/ projects/ghpn-rg/document/draft-ggf-ghpn-net\%issues-0/en/1/ draft-ggf-ghpn-netissues-1.pdf

[3] P. Vicat-Blanc/Primet, "High performance grid networking in the datagrid project," special issue Future Generation Computer Systems, vol. 19, pp. 199-208, Jan. 2003.

[4] A. Chervenak, I. Foster, C. Kesselman, C. Salisbury, and S. Tuecke, "The data grid: Towards an architecture for the distributed management and analysis of large scientific datasets," Journal of Network and Computer Applications, vol. 23, pp. 187200, 2001.

[5] S. Floyd and V. Jacobson, "Link-sharing and resource management models for packet networks," IEEE/ACM Transaction on Networking, vol. 3, pp. 365-386, Aug. 1995.

[6] I. T. Foster, M. Fidler, A. Roy, V. Sander, and L. Winkler, "Endto-end quality of service for high-end applications," Computer Communications, vol. 27, no. 14, pp. 1375-1388, 2004.

[7] T. Roblitz, F. Schintke, and A. Reinefeld, "From clusters to the fabric: the job management perspective," in Proc. IEEE the International Conference on Cluster Computing, 2003, pp. 468473.

[8] K. Ranganathan and I. Foster, "Decoupling computation and data scheduling in distributed data-intensive applications," in Proc. IEEE the 11th Symposium on High Performance Distributed Computing(HPDC'02), July 2002, pp. 352-358.

[9] K. Czajowski, I. Foster, and C. Kesselman, "Resource coallocation in computational grids," in Proc. IEEE the eighth International Symposium on High Performance Distributed Computing, Aug. 1999, pp. 219-228.

[10] H. Dail, F. Bern, and H. Casanova, "A decoupled scheduling approach for grid application development environment," International Journal Parallel and Distributed Systems, vol. 63, pp. 505-524, 2003.

[11] The grid 5000 project. [Online]. Available: http://www.grid5000. org/

[12] L. L. Smarr, A. A. Chien, T. Defanti, J. Leigh, and P. M. Papadopoulos, "The optiputer," Communications of the ACM special issue: blueprint for the future of high-performance networking, vol. 46, pp. 58-67, Nov. 2003.

[13] M. R. Garey and D. S. Johnson, Computers and Intractability, a Guide to the Theory of NP-Completeness. W.H. Freeman and Company, 1979.

[14] L. Marchal, P. Primet, Y. Robert, and J. Zeng, "On network resource scheduling in grid networking," INRIA - ENS/LIP, Lyon, France, Tech. Rep., 2005.

[15] V. Firoiu, J. L. Boudec, D. Towsley, and Z. Zhang, "Theories and models for internet quality of service," Proceedings of the IEEE, vol. 90, pp. 1565-1591, Sept. 2002.

[16] C. Albuquerque, B. Vickers, and T. Suda, "Network border patrol: Preventing congestion collapse and promoting fairness in the internet," IEEE Transactions on Networking, vol. 12, pp. 173186, Feb. 2004.

[17] L. Burchard, H.-U. Heiss, and C. A. F. D. Rose, "Performance issues of bandwidth reservations for grid computing," in Proc. IEEE the 15th Symposium on Computer Architecture and High Performance Computing (SBAC-PAD'03), Nov. 2003, pp. 82-90. 